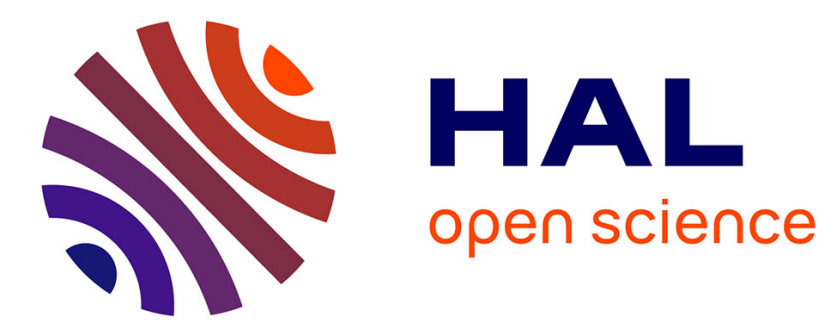

\title{
Multiplexeurs numériques haut débit sur arséniure de gallium pour des applications au-delà de 1,5 Gbit/s
}

\author{
J. Kamdem, M. Le Rouzic, C. Thébault
}

\section{To cite this version:}

J. Kamdem, M. Le Rouzic, C. Thébault. Multiplexeurs numériques haut débit sur arséniure de gallium pour des applications au-delà de 1,5 Gbit/s. Revue de Physique Appliquée, 1988, 23 (7), pp.1229-1242. 10.1051/rphysap:019880023070122900 . jpa-00245935

\section{HAL Id: jpa-00245935 https://hal.science/jpa-00245935}

Submitted on 1 Jan 1988

HAL is a multi-disciplinary open access archive for the deposit and dissemination of scientific research documents, whether they are published or not. The documents may come from teaching and research institutions in France or abroad, or from public or private research centers.
L'archive ouverte pluridisciplinaire HAL, est destinée au dépôt et à la diffusion de documents scientifiques de niveau recherche, publiés ou non, émanant des établissements d'enseignement et de recherche français ou étrangers, des laboratoires publics ou privés. 


\title{
Multiplexeurs numériques haut débit sur arséniure de gallium pour des applications au-delà de $1,5 \mathrm{Gbit} / \mathrm{s}$
}

\author{
J. Kamdem, M. Le Rouzic et C. Thébault \\ C.N.E.T. Lannion, LAB/MER/MLS, route de Trégastel, B.P. 40, 22301 Lannion Cedex, France
}

(Reçu le 27 octobre 1987, révisé le 10 décembre 1987, accepté le 5 janvier 1988)

\begin{abstract}
Résumé. - Deux types de circuits intégrés GaAs de multiplexage haut débit (multiplexeurs à rang fixe et à rang programmable sur 2, 3 ou 4 entrées), capables de fournir des débits numériques jusqu'à 1,9 Gbit/s, ont été conçus et réalisés en logique BFL à grille de $1 \mu \mathrm{m}$. On présente ici la méthode de conception mise en œuvre, et les performances des circuits obtenus. L'architecture de ces multiplexeurs est basée sur des compteurs de type Johnson, sans logique d'établissement de cycle, utilisant des bascules RSTT̄ maître-esclave. Une particularité apportée à ce compteur, consistant à intégrer une partie de la logique de commande dans la bascule du premier étage, a permis le gain d'un temps de propagation de porte sur le temps de boucle du circuit de division. De complexité moyenne (342 à 648 composants actifs par puce), les multiplexeurs ont été simulés en régime non linéaire, selon une méthode de découpage du circuit en blocs quasi indépendants. L'opération de fonderie a été assurée par Thomson/DAG. Les principaux résultats expérimentaux font apparaître une consommation moyenne de moins de $1 \mathrm{~W}$ par puce, et des fréquences maximums de multiplexage variant de 1,3 à $2 \mathrm{GHz}$.
\end{abstract}

\begin{abstract}
High speed MSI GaAs I. C multiplexers operating up to a $1.9 \mathrm{Gbit} / \mathrm{s}$ bit rate have been designed and fabricated in the BFL logic family, using $1 \mu \mathrm{m}$ gate length MESFET technology. Two types of multiplexers have been developed : a fixed version with 2 inputs and programmable versions, 2, 3 or 4 inputs. The design method and the experimental performances of the fabricated chips are presented. The circuits were based on a common architecture including Johnson counters designed with complementary clocked master-slave flipflops. By inserting one half of the control logic into the first stage flip-flop, the loop transit time was reduced by one gate propagation delay. The multiplexers were simulated in the time domain non-linear analysis by using a method which separates the circuit into different quasi independent functional blocks. The Thomson/DAG foundry process was used. The major experimental results show a power consumption around $1 \mathrm{~W}$ and maximum multiplexing frequencies which vary between 1.3 and $2 \mathrm{GHz}$.
\end{abstract}

\section{Introduction.}

Les systèmes de transmission numérique de débit supérieur au Gbit/s vont connaître dans les prochaines années, un développement industriel important sous l'influence de plusieurs facteurs :

i) l'accroissement des capacités requises du fait de l'introduction de nouveaux services de télécommunication ; notamment les futurs réseaux de vidéocommunication ;

ii) les critères économiques qui tendent à prouver qu'un support de transmission doit être utilisé au maximum de sa capacité ;

iii) l'existence d'un support de transmission très large bande ; la fibre optique monomode.
Il s'ouvre ainsi un domaine d'application pour les circuits numériques ultra rapides, domaine dans lequel, malgré la concurrence actuelle avec le silicium [18], les circuits intégrés à base du MESFET GaAs présentent les qualités potentielles de rapidité et de consommation requises pour de telles applications industrielles $[1,2]$. Après les études de faisabilité de ces dernières années sur les capacités du C.I. GaAs à permettre la moyenne ou la haute intégration [12-14], les intérêts actuels tendent vers la conception de tels circuits pour des applications effectives, tant en transmission au-delà du Gbit/s que dans les ordinateurs de très haute rapidité.

Nous présentons dans cet article, une méthode de conception et les performances d'un ensemble de 
circuits intégrés GaAs de multiplexage haut débit, conçus dans une architecture de complexité moyenne, en logique BFL, et fonctionnant à des fréquences d'horloge supérieures à $1,5 \mathrm{GHz}$. Deux types de circuits ont été développés, incluant deux versions d'un multiplexeur à nombre d'entrées programmable de 2 à 4 , et une version à 2 entrées fixes. Des circuits similaires ont déjà été réalisés avec succès dans diffférentes familles logiques GaAs dont la BFL, la DCFL/SBFL [15], [16], la SCFL [17], [19].

On décrit tout d'abord l'architecture des circuits et la méthode de conception utilisée. L'outil de base est une bibliothèque de fonctions logiques $\mathrm{BFL}$, basée sur des cellules standards fournies par Thomson/DAG, que nous avons modélisée dans ASTEC3 autour d'un modèle non linéaire de MESFET GaAs $[9,11]$, et qui a été adapté au régime de commutation $[8,10]$ et aux caractéristiques de la technologie du fondeur. Une analyse par simulation, des aléas de fonctionnement liés au régime des hautes vitesses, a été effectuée sur les opérateurs logiques utilisés. On présente ensuite les résultats de la caractérisation dynamique et les performances des circuits montés en boîtier.

\section{Fonctionnalité des circuits.}

Ces circuits sont destinés aux étages finals d'un équipement de multiplexage temporel pour système de transmission à haut débit sur fibre optique [3]. La figure 1 présente un exemple d'architecture d'un tel équipement dans lequel, l'information à transmettre se présente sous la forme de $\boldsymbol{n}$ signaux synchrones au débit de $140 \mathrm{Mbit} / \mathrm{s}$. Après un codage de type $n \mathrm{~B} m \mathrm{~B}$, nécessaire pour des raisons liées à l'exploitation du système, l'information se retrouve sous la forme de $m$ signaux à $140 \mathrm{Mbit} / \mathrm{s}(n=16$ et $m=18$ dans l'exemple choisi). Un multiplexage temporel permet ensuite de former le signal numérique modulant la diode laser.

Sur la figure 1 , ce multiplexage est effectué en trois étapes, chacune faisant intervenir un niveau technologique différent: ECL sur silicium pour l'étage $420 \mathrm{Mbit} / \mathrm{s}$, circuits intégrés sur GaAs semispécifiques pour l'étage 1,2 Gbit/s (c'est le domaine d'utilisation des circuits présentés ici) et circuit

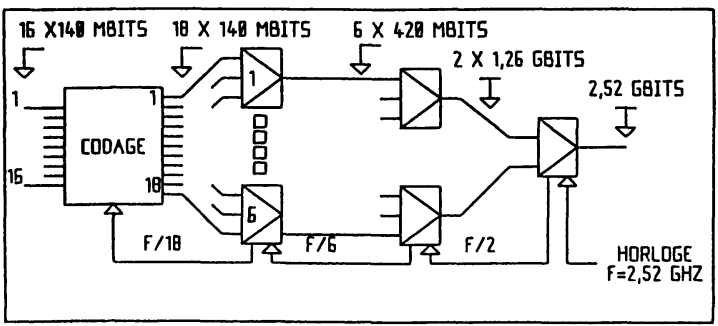

Fig. 1. - Architecture d'un système $16 \times 140 \mathrm{Mbit} / \mathrm{s}$.

[16 $\times 140 \mathrm{Mbit} / \mathrm{s}$ system architecture.] intégré GaAs spécifique pour l'étage le plus rapide. Cette brève description permet de préciser les fonctions requises au niveau des circuits des étages les plus rapides :

- multiplexage élément binaire par élément binaire ;

- base de temps intégrée à la logique de multiplexage ;

- possibilité de synchroniser entre elles les bases de temps de plusieurs circuits pour créer un multiplexeur de rang plus élevé ;

- rang de multiplexage programmable pour étendre le domaine d'utilisation.

Dans la suite on décrira essentiellement un multiplexeur à 2 entrées répondant aux deux premiers critères, et 2 versions d'un multiplexeur programmable à 2,3 ou 4 entrées présentant toutes les fonctionnalités précédentes.

\section{Conception des multiplexeurs.}

3.1 ARCHITECTURE DES CIRCUITS. - L'ensemble des multiplexeurs a été conçu sur une architecture commune dont le schéma est représenté sur la figure 2. Elle est constituée de 4 blocs fonctionnels travaillant en mode synchrone sous le contrôle de deux horloges $H_{1}$ et $H_{2}$ déphasées mais de même fréquence :

i) Le premier bloc fonctionnel est un compteur synchrone de type Johnson à initialisation automati-

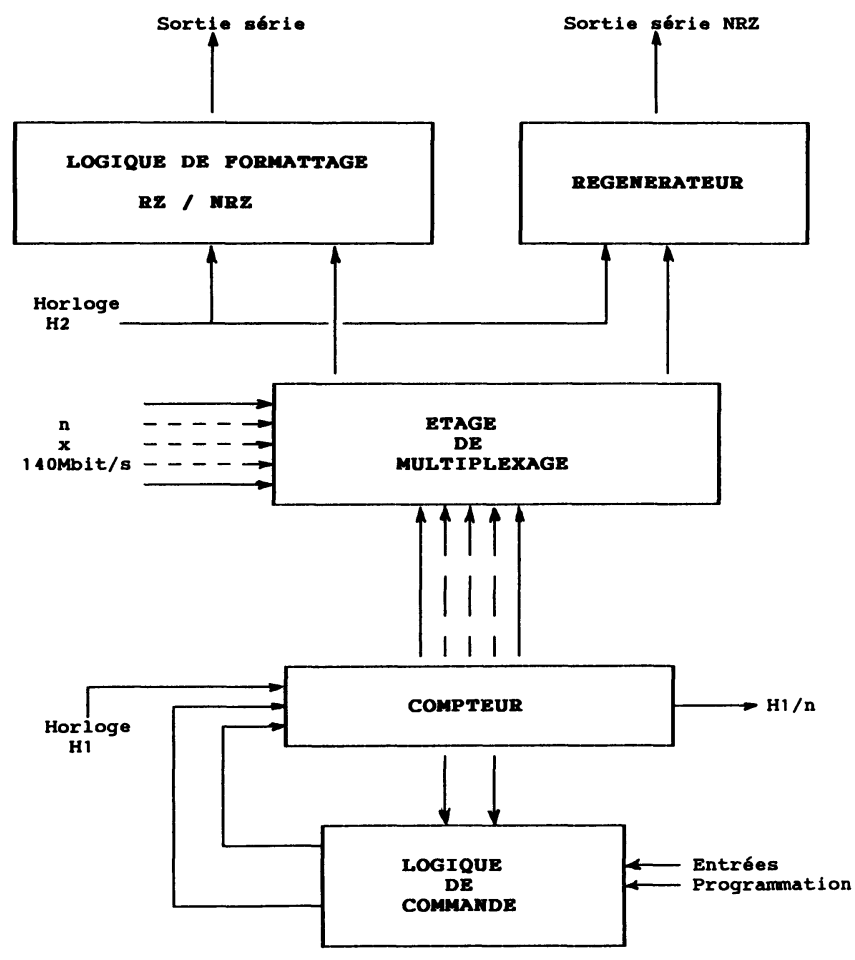

Fig. 2. - Architecture générale des multiplexeurs.

[General block diagram of multiplexers.] 


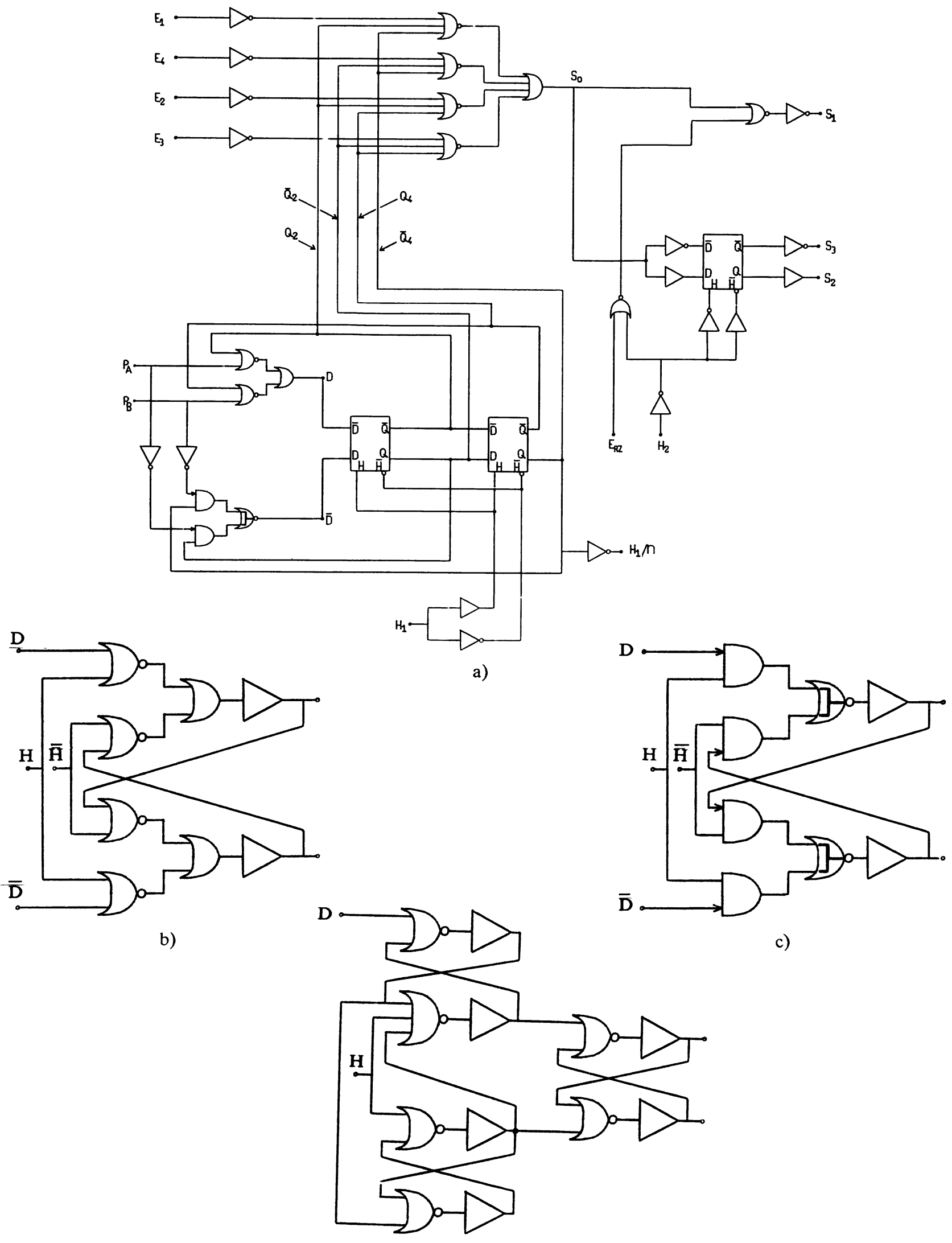

d)

Fig. 3. - (a) Logigramme du multiplexeur programmable; (b) bascule RSTT à portes NOR/OR ; (c) bascule RSTT à portes AND/NOR; (d) bascule D à 6 portes NOR.

[Logic diagrams of programmable multiplexers (a), complementary clock RS latch using NOR/OR (b) or AND/NOR gates (c), and a 6 buffered NOR gate D type flip-flop (d).] 
que à bascule RSTT̄ maître-esclave (que nous noterons par la suite RST $\bar{T} M / E$ ), dont le rang de division $n$ est programmable par 2, 3 ou 4 pour les multiplexeurs de la première version et à nombre d'entrées fixe pour ceux de la seconde version. La logique de commande est un sous-circuit combinatoire chargé de générer les excitations secondaires nécessaires aux compteurs, à partir des variables internes du circuit et du vecteur de données de programmation (voir Fig. 3a).

Le compteur délivre un signal (noté $H_{1} / n$ ), destiné à piloter les étages de multiplexage de rang inférieur, travaillant à la fréquence $H_{1} / n$ (voir Fig. 1).

ii) Les $n$ signaux à traiter arrivent en parallèle sur le second bloc fonctionnel qui est un étage combinatoire de multiplexage, intégrant la logique de détection de la fenêtre de transmission.

iii) Le troisième bloc fonctionnel est un circuit de décision (bascule D ou RSTT $M / E$ ) actionné par $\mathrm{H}_{2}$, qui effectue la régénération du signal série, sans retour à zéro (NRZ).

iv) Le dernier bloc est simplement constitué de 2 portes NOR qui réalisent le formattage du signal multiplexé $S_{0}$, soit en RZ (retour à zéro), soit en NRZ, selon la valeur de l'entrée $E_{\mathrm{rz}}$ (Fig. 3a).

3.2 ANAlyse logiQue ET FONCTIONNELlE. - Le logigramme de la figure $3 a$ représente le multiplexeur programmable dans son ensemble, où $\mathbf{E}=\left(E_{1}, E_{2}, E_{3}, E_{4}\right)$ désigne le vecteur de données, et $\mathbf{P}_{\mathrm{p}}=\left(\boldsymbol{P}_{\mathrm{A}}, \boldsymbol{P}_{\mathrm{B}}\right)$, le vecteur de programmation. Sur le principe de ce schéma, deux versions de ce multiplexeur, se distinguant par le type de circuit de décision adopté ont été conçus.

La version 1 comporte la bascule RSTT à demiregistres NOR/OR (Fig. 3b) au niveau du compteur et une bascule D à 6 portes NOR (Fig. 3d) dans le bloc de régénération, tandis que la version 2 ne comprend que la bascule RST $\overline{\mathrm{T}}$ à demi-registres AND/NOR (Fig. 3c).

Comme l'indique le schéma de principe de la figure 3a, le compteur programmable s'identifie à un circuit séquentiel, caractérisé par :

- 2 excitations primaires $P_{\mathrm{A}}$ et $P_{\mathrm{B}}$;

- 2 excitations secondaires $D$ et $Q_{2}$;

- 2 variables internes $Q_{2}$ et $Q_{4}$;

$\bar{Q}_{4}$.

- et 4 variables de sorties $Q_{2}, \bar{Q}_{2}, Q_{4}$ et

Le fonctionnement de ce circuit est entièrement décrit d'une part, par les équations définissant les états présents à l'instant $t$ sur les nœuds des excitations secondaires :

$$
\begin{aligned}
& D(t)=\overline{Q_{2}(t)+P_{\mathrm{A}}}+\overline{Q_{4}(t)+P_{\mathrm{B}}} \\
& Q_{2}(t)=Q_{2}(t) \quad \text { l'état de } Q_{2} \text { reste inchangé }
\end{aligned}
$$

et d'autre part, par les relations qui engendrent les états suivants aux instants $t+\delta t$ sur les variables internes :

$$
\begin{aligned}
& Q_{2}(t+\delta t)=D(t) \\
& Q_{4}(t+\delta t)=Q_{2}(t) .
\end{aligned}
$$

En associant le code de la figure 4a aux 4 états possibles du compteur, les expressions (1) à (4) permettent d'établir la table des excitations secondaires sur $D$ et $Q_{2}$ (Fig. 4b), et celle des états en sortie sur $Q_{2}, \bar{Q}_{2}, Q_{4}$ et $\bar{Q}_{4}$ (Fig. $4 \mathrm{c}$ ).

A chaque valeur du vecteur $\left(P_{\mathrm{A}}, P_{\mathrm{B}}\right)$, correspond un cycle différent du compteur, correspondant aux comptages successifs par 4,3 et 2 . Seul l'état $(1,1)$ pour lequel le circuit est dans un état figé, est interdit. Les diagrammes des transitions déduits de la table des excitations secondaires pour chaque rang de division sont donnés sur les figures $5 \mathrm{a}$ à c. On note que dans chaque cas, il y a récupération automatique du cycle, et qu'il n'y a pas d'ambiguïté sur le sens de comptage, ceci quel que soit l'état initial du compteur.

En additionnant les points à l'état 0 sur la table des sorties (Fig. 4c), on obtient les expressions littérales des 4 variables, $q_{1}, q_{2}, q_{3}, q_{4}$ contenant chacun la fenêtre associée à une voie de multiplexage, sous les formes:

$$
\begin{aligned}
& q_{1}=Q_{2}+\bar{Q}_{4} \\
& q_{2}=Q_{2}+Q_{4} \\
& q_{3}=\bar{Q}_{2}+Q_{4} \\
& q_{4}=\bar{Q}_{2}+\bar{Q}_{4} .
\end{aligned}
$$

Pour chaque vecteur de programmation, les opérations (5) à (8) provoquent la rotation d'un niveau 0 sur les entrées de l'étage de multiplexage, permettant la sélection de la voie de transfert. La figure $4 d$ rassemble les états de sortie sur les variables $q_{1}$ à $q_{4}$.

La fréquence maximum de division dépend pour une large part du temps mis par le circuit à l'élaboration du nouvel état présent aux entrées $D$ et $\overline{\mathrm{D}}$ du premier étage du compteur, compte tenu de l'expression (1). Afin de minimiser ce délai, nous avons dédoublé l'entrée $\mathrm{D}$ par l'addition d'un opérateur supplémentaire, de sorte que la valeur de $D(t)$ est élaborée dans le premier demi-registre de cet étage, ce qui permet de supprimer une couche logique dans les boucles de retour. La figure 6a illustre cette amélioration, pour les compteurs de la version 2 où 5 opérateurs AND constituent les entrées de la première bascule, tel que :

$Q_{1}(t+\delta t)=[\overline{D(t)}+\overline{H(t)}] \cdot\left[H(t)+Q_{1}(t)\right]$

Le schéma électrique de ce demi-registre modifié est représenté sur la figure $6 \mathrm{~b}$, où les portes AND sont 


\begin{tabular}{l|ll}
$\begin{array}{l}\text { Etat du } \\
\text { compteur }\end{array}$ & $Q_{1}$ & $Q_{2}$ \\
\hline 1 & 0 & 0 \\
2 & 1 & 0 \\
3 & 1 & 1 \\
4 & 0 & 1
\end{tabular}

a)

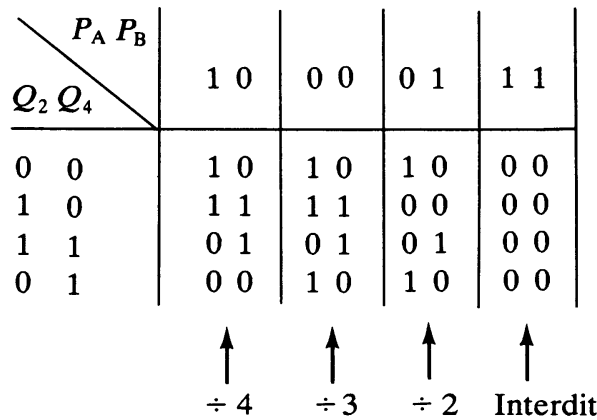

Vecteur $\left(D, Q_{2}\right)$

b)

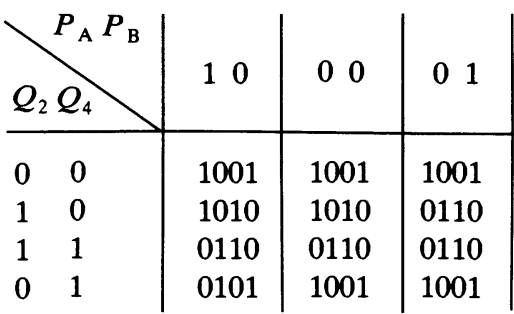

Vecteur $\left(Q_{2}, \bar{Q}_{2}, Q_{4}, \bar{Q}_{4}\right)$

c)

\begin{tabular}{|c|c|c|c|c|}
\hline 2 & & 10 & 00 & $\begin{array}{ll}01 \\
\end{array}$ \\
\hline 0 & 0 & 1101 & 1101 & 1101 \\
\hline 1 & 0 & 1110 & 1110 & 0111 \\
\hline 1 & 1 & 0111 & 0111 & 0111 \\
\hline 0 & 1 & 1011 & 1101 & 1101 \\
\hline
\end{tabular}

Vecteur $\left(q_{1}, q_{2}, q_{3}, q_{4}\right)$

d)

Fig. 4. - (a) Code associé aux états du compteur programmable; (b) table des excitations secondaires, $\left(D, Q_{2}\right)$ aux différents cycles de comptage ; (c) table des états en sortie sur le vecteur $\left(Q_{2}, \bar{Q}_{2}, Q_{4}, \bar{Q}_{4}\right)$; (d) table des états en sortie sur le vecteur $\left(q_{1}, q_{2}, q_{3}, q_{4}\right)$.

[Table of states of the programmable counter.]

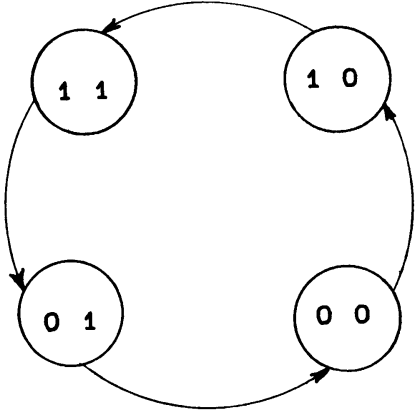

a)

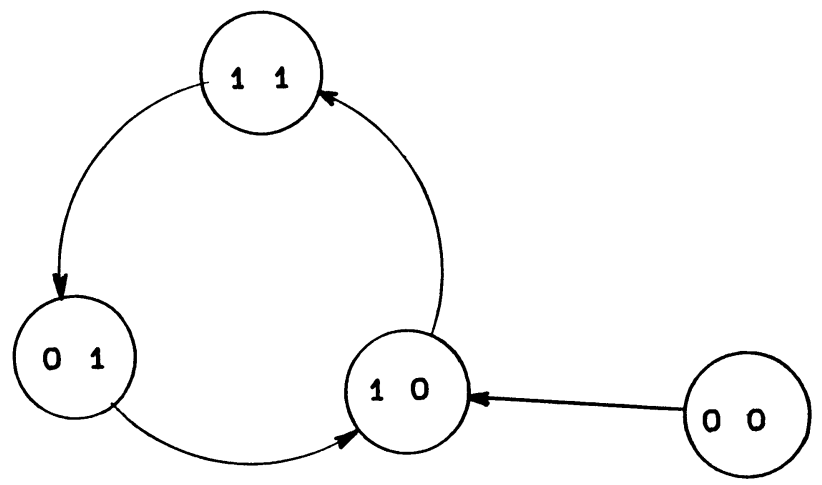

b)

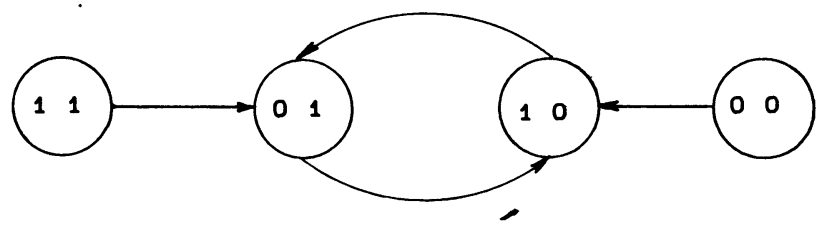

c)

Fig. 5. - Diagrammes des transitions du compteur programmable. (a) Division par 4 ; (b) division par 3 ; (c) division par 2.

[Transition diagrams of the programmable counter.]

réalisées par des transistors bi-grilles. Les capacités parasites associées ont été évaluées sur les dessins de masque.

3.3 Simulation DES MULTIPLEXEURS EN RÉGIME TEMPOREL. - La simulation des multiplexeurs a été effectuée dans le domaine temporel dans ASTEC3, à partir d'une bibliothèque de fonctions BFL que nous avons modélisée sur la base de cellules standards et des caractéristiques de la technologie $1 \mu \mathrm{m}$ de Thomson/DAG, en employant un modèle de MESFET GaAs précédemment validé à $300 \mathrm{~K}$ dans le domaine non linéaire $[4,5,9]$. Pour cela, une analyse par simulation du comportement des sousfonctions BFL lorsque la période d'horloge est 

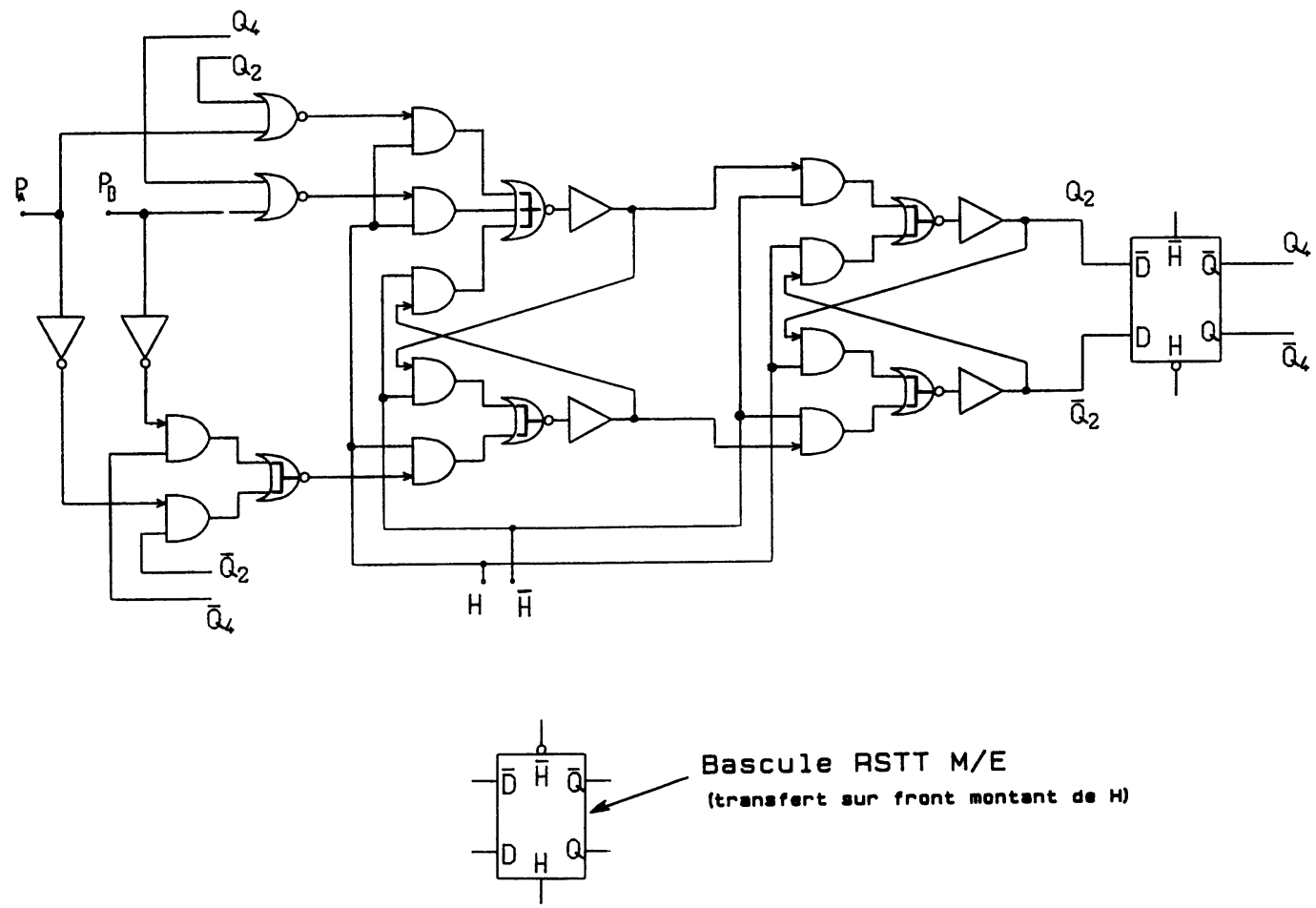

a)

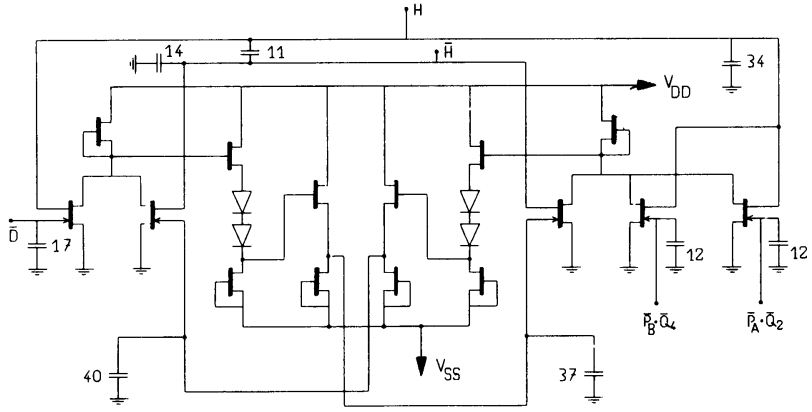

b)

Fig. 6. - (a) Détail du demi-registre modifié, de la bascule d'entrée du compteur programmable à portes AND/NOR. La donnée $D(t)$ est élaborée à l'intérieur de ce demi-registre, ce qui permet la suppression d'une couche logique dans la logique de commande; (b) schéma électrique du demi-registre modifié. Capacités évaluées sur layout (en fF).

[(a) Logic diagram of the programmable registers designed with AND/NOR gates ; (b) circuit schematic diagram of the first RS latch.]

voisine du temps de propagation a été effectuée ; il s'agit notamment de la bascule RSTT̄ M/E qui est très rapide, mais dont les risques de perte d'information [6], sont accentués lorsque la période d'horloge est voisine du temps de propagation des portes.

L'exemple de résultats de simulation des figures $7 \mathrm{a}$ et $\mathrm{b}$, décrit les conséquences du passage simultané de $H$ et $\bar{H}$ au-dessus du seuil de commutation, sur une bascule RSTT $\mathrm{M} / \mathrm{E}$ à portes NOR/OR dont le transfert de l'information a lieu sur le front descendant de $H$. Le paramètre $t_{\mathrm{r} 1}$ définit la durée pendant laquelle les 2 horloges se croisent à 1 (Fig. 7a).
Comme l'indique la figure $7 \mathrm{~b}$, plus $t_{\mathrm{r} 1}$ est important (sans toutefois dépasser la marge de fonctionnement qui est définie par le temps de propagation $T_{\mathrm{pd}}$ de l'ensemble NOR/OR/BUFFER), plus les creux sur le niveau logique en sortie, traduisant le début de perte d'information sont profonds. Dans le même temps, les fronts descendants qui correspondent à la lecture du « 0 » à l'intérieur de la bascule, se déplacent en fonction de la durée de croisement $t_{\mathrm{r} 1}$, tandis que le front montant (lecture d'un « $\left.1 »\right)$ en est indépendant. Ainsi la variation du rapport cyclique du signal est une fonction exclusive du front 


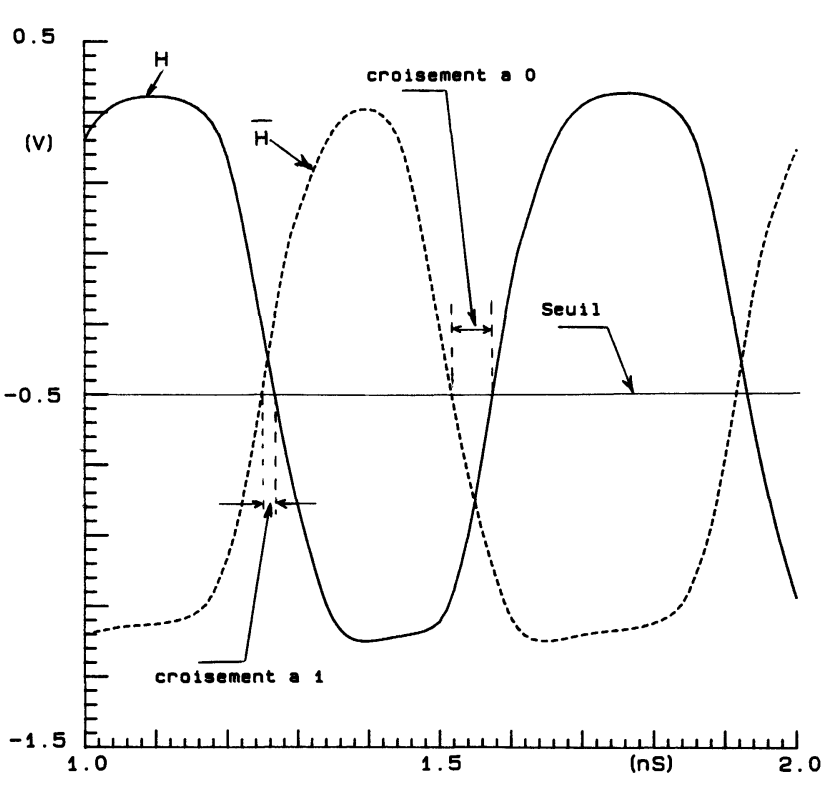

a)

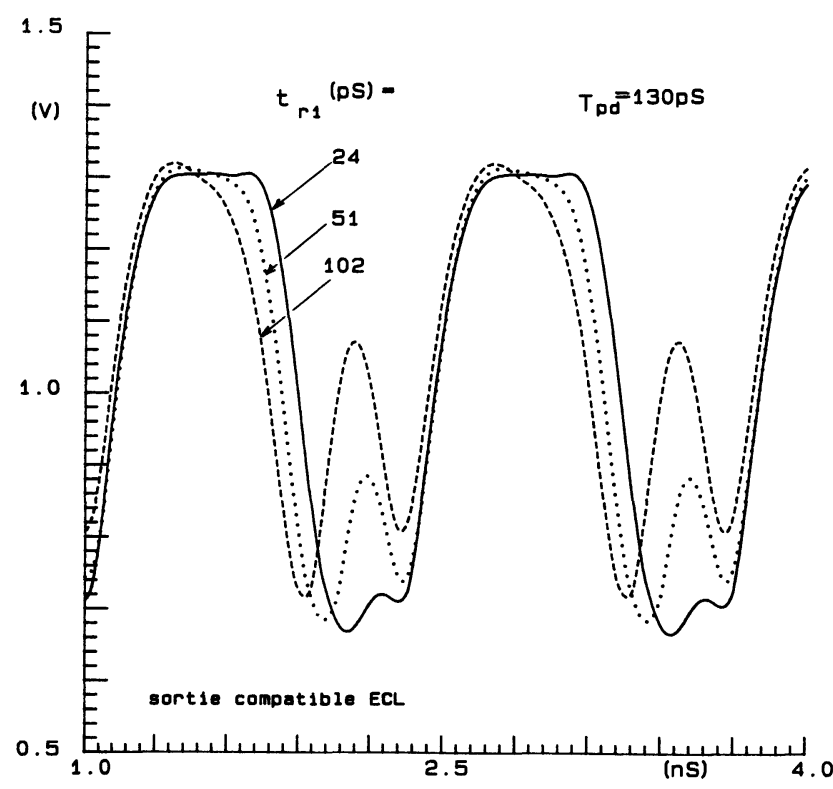

b)

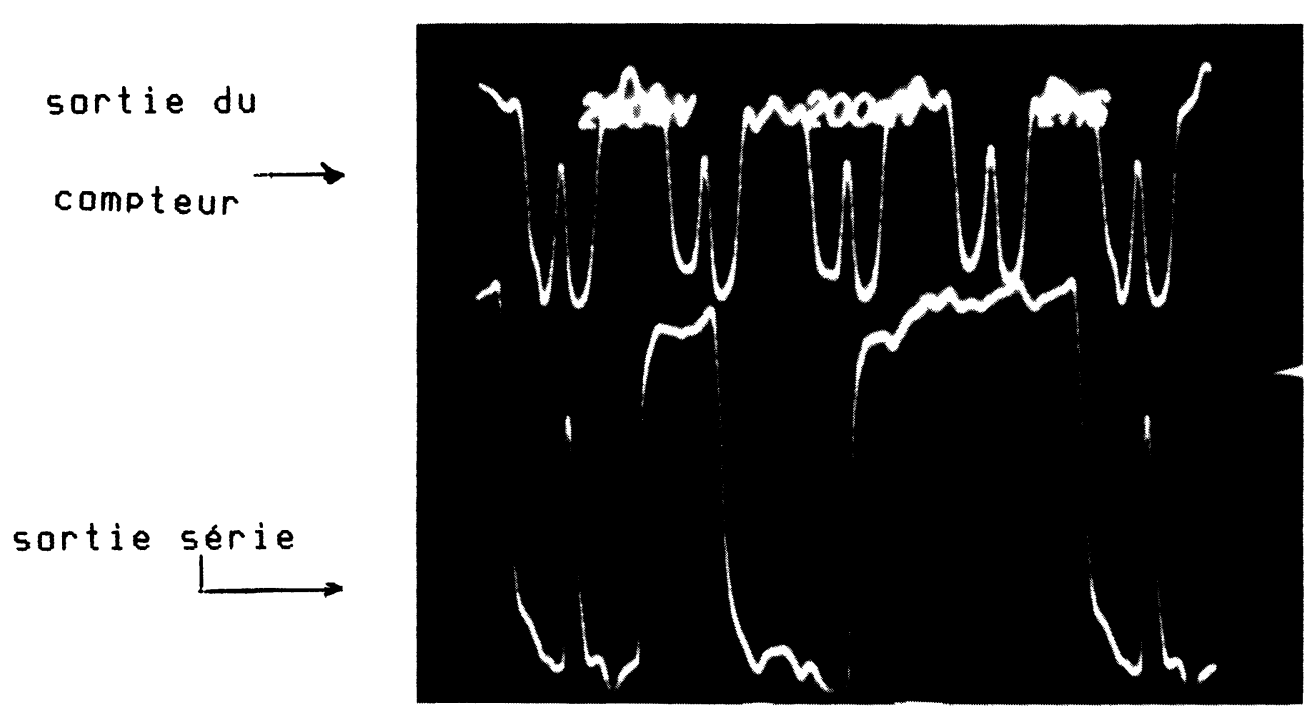

c)

Fig. 7. - Mise en évidence des aléas provoqués sur le signal de sortie d'une bascule RST $\bar{T}$ M/E à portes NOR/OR par le passage simultané des horloges complémentaires au-dessus du seuil de commutation. (a) Formes d'ondes des horloges complémentaires ; (b) variations de la profondeur des creux traduisant la dégradation de l'état logique, en fonction de la durée $t_{\mathrm{r} 1}$ de croisement des horloges à 1 ; (c) photographie de ces aléas sur un multiplexeur à 2 entrées : trace du haut $=$ sortie du compteur ; trace du bas $=$ sortie série ; (d) photographie du signal délivré par cette bascule (trace du bas) connecté en diviseur par 2 , et pilotée par une horloge (trace du haut) dont la polarisation continue est telle que le déphasage entre $H$ et $\bar{H}$ est instable. Seuls les fronts montants sont stables.

[Computed waveforms showing parasitic effects introduced on the output signal of a master-slave flip-flop (including NOR/OR gates) when both $H$ and $\bar{H}$ are high. Photo (c) and (d) present the corresponding measured waveforms.]

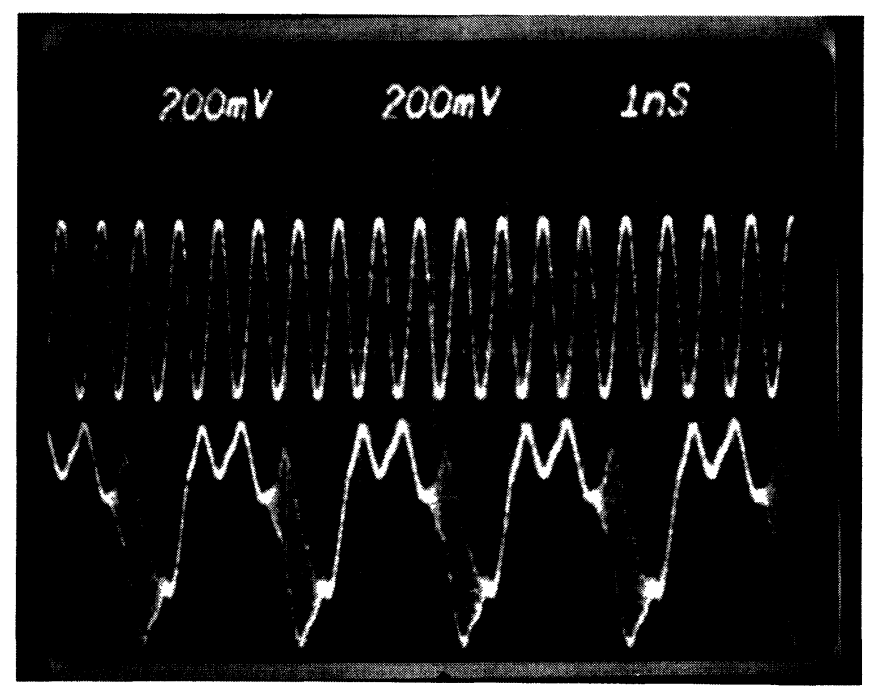

d) 
descendant. Dans la pratique, l'une des conséquences de ces résultats, pour un circuit, est qu'une variation parasite de phase entre $H$ et $\bar{H}$ se répercutera sur le signal de sortie sous la forme d'une instabilité au niveau du front descendant.

Par la suite, nous reviendrons sur ces phénomènes au cours de la caractérisation expérimentale.

Compte tenu de la complexité des circuits étudiés (de 342 à 648 composants actifs), une méthode de simulation [8] permettant de minimiser le temps calcul sur ordinateur et de résoudre le problème de place mémoire par rapport à ASTEC3 a été utilisée. Elle consiste à séparer le circuit logique en blocs fonctionnels quasi indépendants et à les simuler séparément, après avoir remplacé les circuits environnants par des sortances équivalentes, exprimées en terme de capacités. Pour cela, nous déterminons tout d'abord les sortances ramenées par les autres blocs, sur les nœuds de connexion avec le circuit devant être simulé. Chargé par les valeurs de ces sortances, le circuit est simulé en régime transitoire et les formes d'ondes générées aux nœuds d'accès sont stockées sous forme de tableaux de valeurs qui seront rappelés dans une simulation ultérieure en temps que sources de tension numériques pour les excitations d'un autre bloc fonctionnel. Les impédances internes de ces sources sont alors régénérées par un étage buffer, ou un inverseur, connecté en série. Une bibliothèque de signaux numériques de formes complexes, spécifiques à chacune des sous-fonctions logiques composant le multiplexeur étudié, est ainsi déterminée :

- forme d'onde des horloges complémentaires;

- signaux d'états délivrés par le compteur;

- signaux série délivrés par l'étage de multiplexage.

Les effets de propagation sont négligés compte tenu des faibles longueurs des lignes de connexion (moins de $0,5 \mathrm{~mm}$ ), par rapport aux fréquences mises en jeu (1 à 2,5 GHz). Par suite, les effets de couplage entre métallisations sont assimilés à des capacités localisées entre nœuds, à l'exclusion de tout élément selfique. Ces capacités sont évaluées sur les dessins de masques de chaque circuit.

Les figures $8 \mathrm{a}$ à $\mathrm{d}$ présentent les formes d'ondes délivrées en simulation par le multiplexeur programmable représenté sur la figure $3 \mathrm{a}$, dans les trois modes de fonctionnement possible, à $1,5 \mathrm{GHz}$ de fréquence d'horloge, les valeurs de polarisation étant $V_{\mathrm{DD}}=2,3 \mathrm{~V}$ et $V_{\mathrm{SS}}=-2,2 \mathrm{~V}$.

Les simulations effectuées en fonction de la tension de seuil $V_{\mathrm{T}}$ ont fournit des fréquences maximums théoriques de $1.7 \mathrm{GHz}$ pour $V_{\mathrm{T}}=-0,75 \mathrm{~V}$ et $2,2 \mathrm{GHz}$ pour $V_{\mathrm{T}}=-1 \mathrm{~V}$ sur l'ensemble des circuits.

$\mathrm{Au}$ terme de la phase de conception, un réticule de $5040 \mu \mathrm{m} \times 4850 \mu \mathrm{m}$ a été mis au point. Il contient deux versions du multiplexeur programmable (MUXV1 et MUXV2), un multiplexeur à 2 entrées (MUX2) et des motifs de contrôle du procédé technologique du fondeur.

\section{Réalisation, caractérisation et mise en œuvre.}

4.1 RÉAlisAtion. - Les circuits ont été réalisés par THOMSON/DAG dans une technologie présentant les caractéristiques suivantes : grille de $1 \mu \mathrm{m}$, 2 niveaux de métallisation, couche active implantée, tension de seuil nominale de $-1 \mathrm{~V}$ pour un compromis vitesse/consommation de l'ordre de 200 ps pour $3 \mathrm{~mW}$ (porte NOR à 2 entrées chargée par 2 circuits et $0,5 \mathrm{~mm}$ de connexion). La figure 9 présente la photographie d'un multiplexeur programmable: $1536 \mu \mathrm{m} \times 1032 \mu \mathrm{m}$ pour 289 transistors et 172 diodes. Les circuits ont fait l'objet chez le fondeur d'une caractérisation fonctionnelle basse fréquence sur la plaquette, afin de sélectionner les puces à monter en boîtier.

4.2 CARACTÉRISATION DYNAMIQUE. - Les circuits sont montés dans des boîtiers à 16 accès de type MS16L [7], spécialement étudiés pour les circuits rapides. Pour les tests dynamiques, le boîtier est rapporté sur un circuit imprimé en DUROID portant des lignes d'impédance $50 \Omega$. Des composants passifs de type pavé sont utilisés pour réaliser les adaptations d'impédance et les découplages d'alimentation. Lorsque c'est nécessaire, les valeurs des polarisations de la puce de GaAs peuvent être choisies pour assurer la comptabilité des niveaux logiques avec les étages ECL, dont l'excursion logique est adoptée comme référence.

4.2.1 Tests fonctionnels. - Ces mesures sont effectuées à moyenne fréquence (300 à $600 \mathrm{MHz}$ ) et permettent de valider la conception logique et les dessins d'implantation. Dans le cas du multiplexeur programmable, un vecteur de données $\mathbf{E}$ ayant la configuration suivante est appliqué au circuit :

$$
\mathbf{E}=\left[\begin{array}{c}
E_{1} \\
E_{2} \\
E_{3} \\
E_{4}
\end{array}\right]=\left[\begin{array}{c}
f / 8 \\
0 \\
1 \\
0
\end{array}\right]
$$

$f$ étant la fréquence d'horloge du compteur $\left(H_{1}\right)$. Pour une programmation sur 4 entrées, la sortie série $S_{1}$ multiplexée délivre la séquence numérique représentée sur la figure 10 , où chaque élément binaire a la durée d'une période d'horloge. Sur cette figure, la photographie correspondante a été prise à la fréquence de $600 \mathrm{MHz}$.

4.2.2 Les aléas de la bascule RSTT̄ M/E aux limites de fonctionnement. - Les déformations de l'état logique bas simulées précédemment sur la bascule 


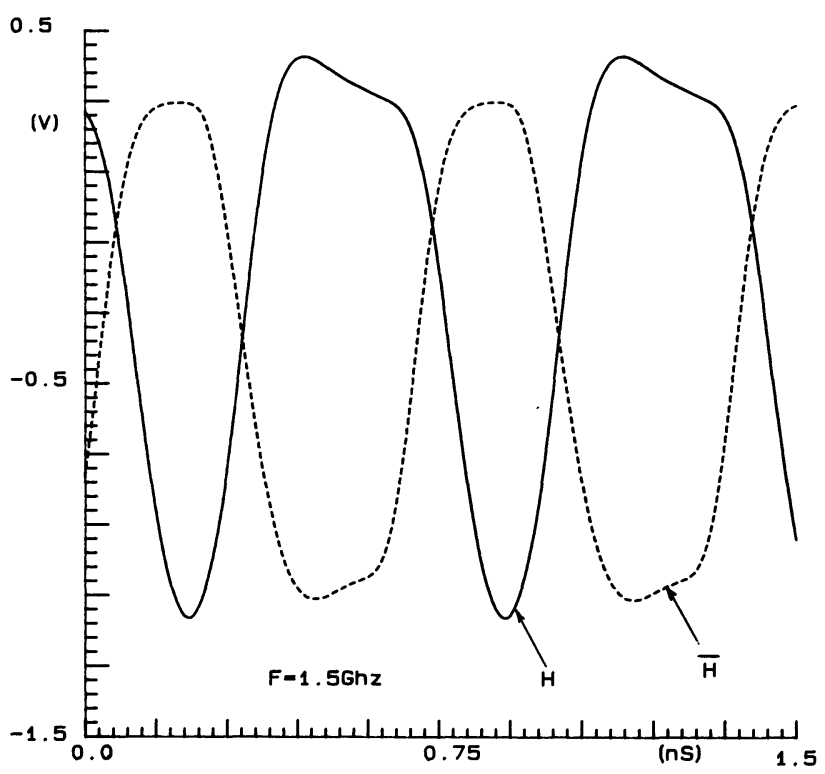

a)

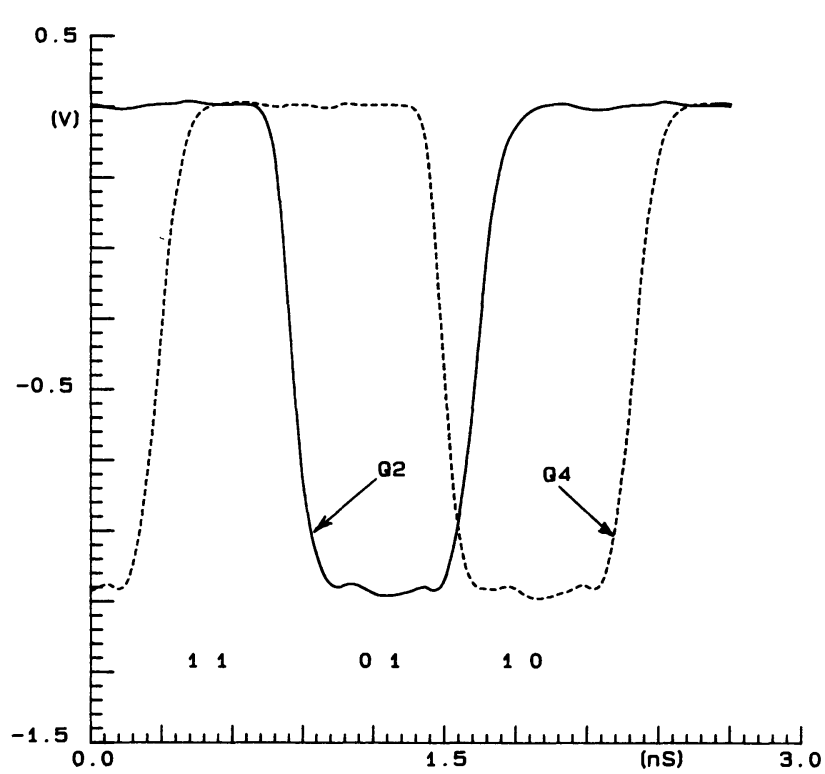

c)

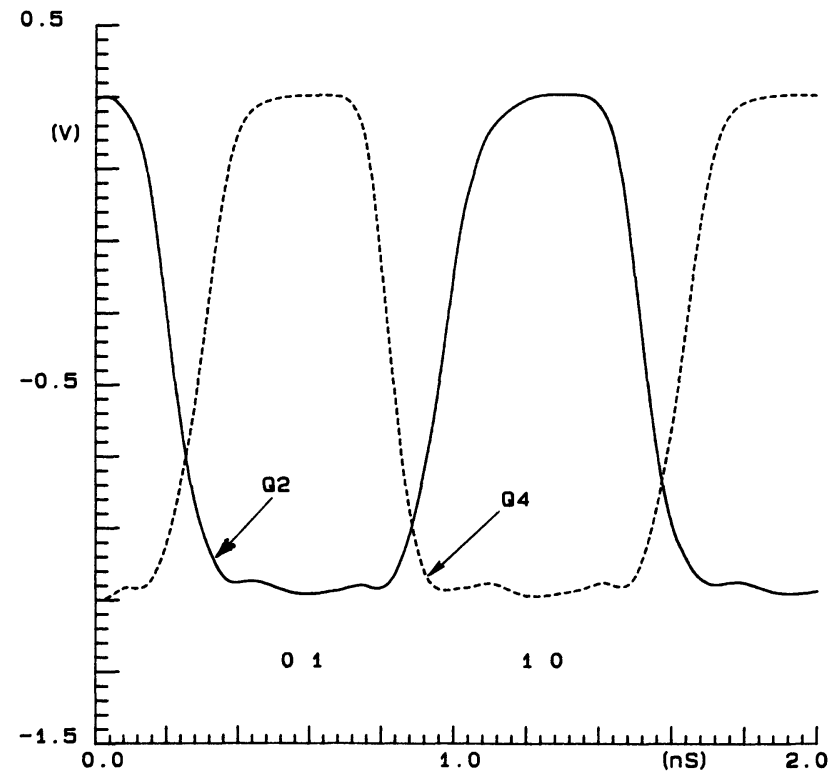

b)

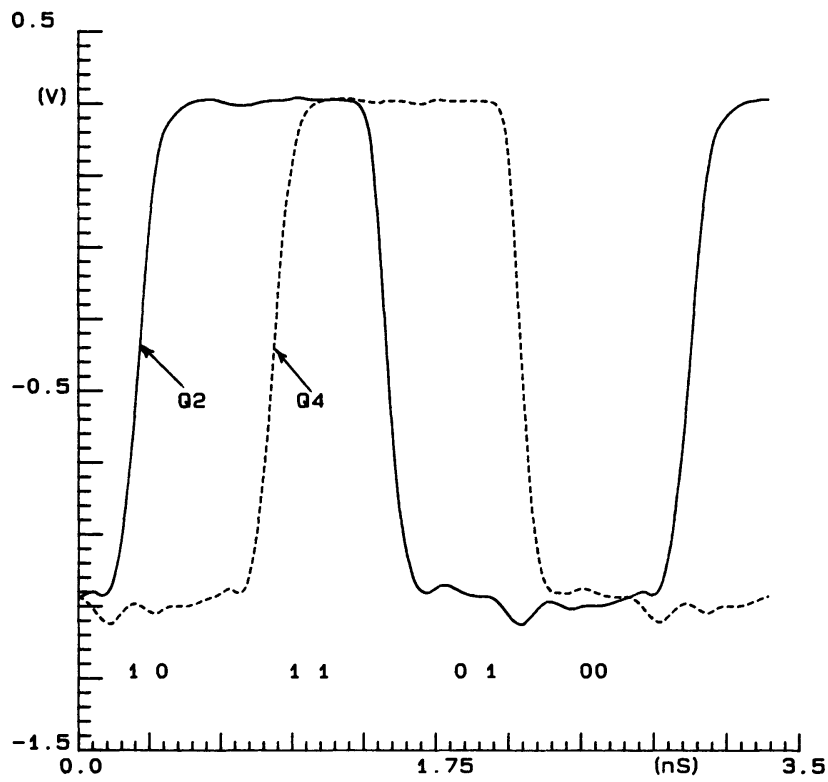

d)

Fig. 8. - Formes d'ondes simulées du compteur programmable représenté sur la figure 3a. En (a), on a le signal horloge à $1,5 \mathrm{GHz}$; en (b), le signal délivré par le compteur en division par 2 , pour $\left(P_{\mathrm{A}}, P_{\mathrm{B}}\right)=(0,1)$; en $(\mathrm{c})$, la division par 3 , pour $\left(P_{\mathrm{A}}, P_{\mathrm{B}}\right)=(0,0)$; enfin en $(\mathrm{d})$, la division par 4 pour $\left(P_{\mathrm{A}}, P_{\mathrm{B}}\right)=(1,0)$.

[Output waveforms of the counter represented in figure 3a.]

RSTT̄ M/E (Fig. 7b), sont mis en évidence sur la photographie de la figure 7c. Ces aléas ont été relevés sur le diviseur par 2 d'un multiplexeur à 2 entrées comportant la bascule à portes NOR/OR, la polarisation externe de l'horloge $H_{1}$ étant telle que $H$ et $\bar{H}$ se croisent au-dessus du seuil de commutation. Pour un système bouclé tel qu'un compteur, où les données sont générées par le circuit lui-même, la cohérence de l'ensemble impose un temps de maintien toujours suffisant entre 2 cycles d'évolution, de sorte que le fonctionnement est moins critique en haute fréquence ; il n'en serait pas de même en transmission de donnée où ces aléas peuvent être à l'origine de pertes totales des informations mémorisées, dès que ces creux traversent le seuil de commutation.

La photographie de la figure $7 \mathrm{~d}$ met en évidence l'instabilité des fronts descendants du signal délivré 


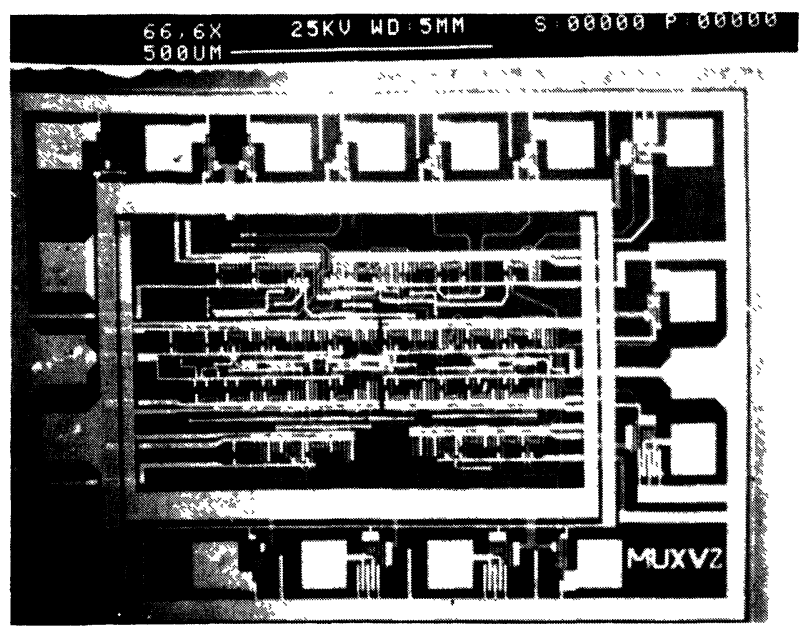

Fig. 9. - Photographie d'une puce du multiplexeur programmable (MUXV2) $(1536 \mu \mathrm{m} \times 1032 \mu \mathrm{m})$.

[Chip microphotograph of a programmable multiplexer.]

par ce type de bascule, lorsque la durée de croisement des horloges à 1 varie. Ce signal a été relevé à $1840 \mathrm{MHz}$, sur le diviseur par 4 d'un multiplexeur
MUXV1 dont le niveau de polarisation de l'horloge principale $H_{1}$ est choisi de sorte que les signaux $H$ et $\bar{H}$ générés se trouvent à cheval sur le croisement à 1 et à 0 . Dans cette situation, le déphasage entre $H$ et $\bar{H}$ devient totalement instable. En accord avec le résultat théorique de la figure $7 \mathrm{~b}$, les fronts montants du signal délivré dans ces conditions par le diviseur restent stables tandis que les fronts descendant fluctuent sur un intervalle temporel qui dans ce cas particulier est d'environ $350 \mathrm{ps}$. L'un des tous premiers intérêts de ces résultats est de montrer que dans les applications où seuls les fronts du signal délivré par le diviseur sont exploités, il est préférable de n'utiliser que les fronts montants, afin d'être à l'abri de perturbations pouvant être liées à des fluctuations de phase entre $H$ et $\bar{H}$. Pour une bascule à transfert sur front montant, ce sont les fronts montants qui seraient instables.

4.2.3 Mesures en fréquence. - Les mesures dynamiques, ont pour but de déterminer les performances des circuits, dans les conditions de fonctionnement ultime en fréquence. Ainsi que le montre la figure 3, les blocs fonctionnels peuvent être testés indépen-
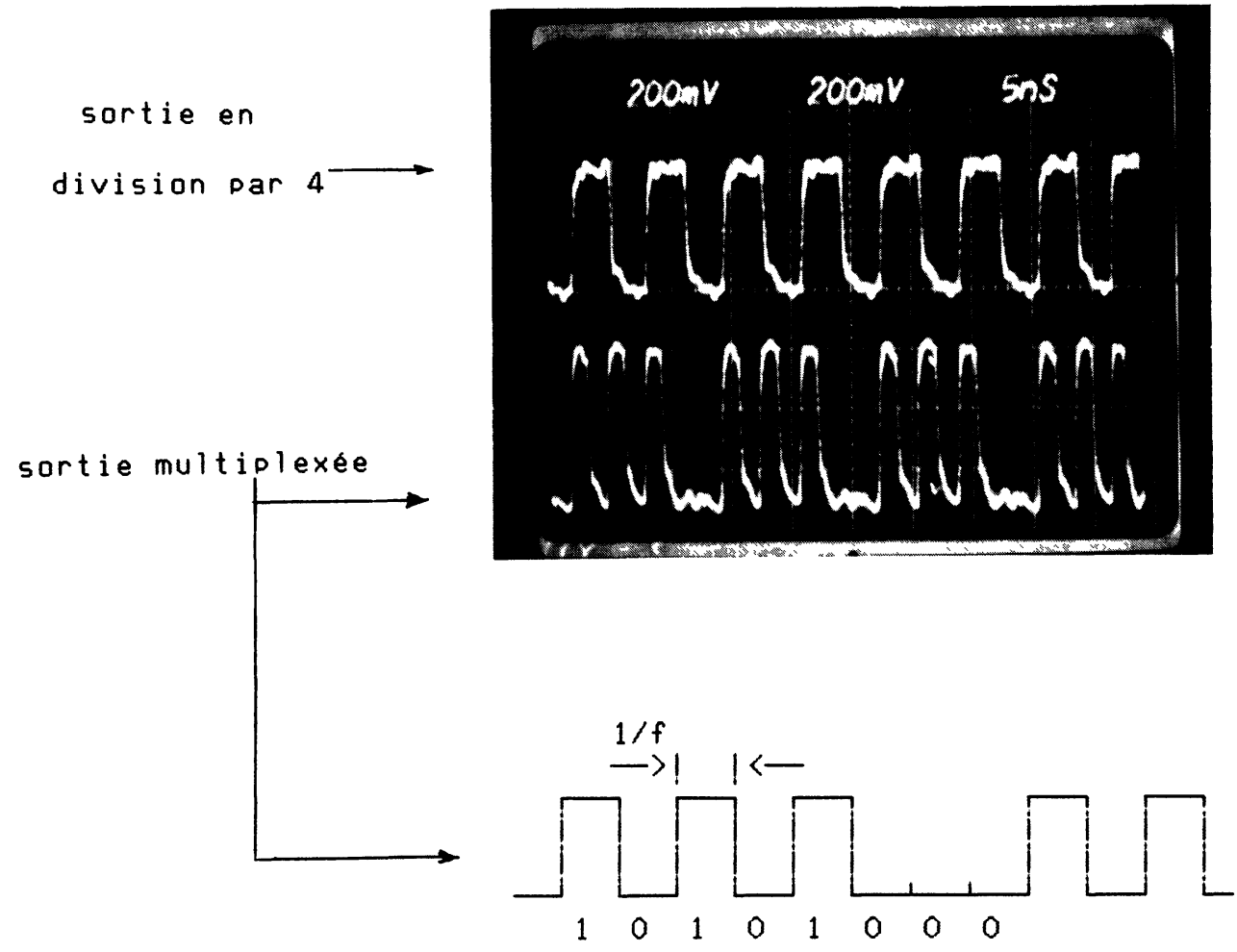

8 BITS

Fig. 10. - Photographie de la séquence délivrée par un multiplexeur programmé sur 4 entrées, en test fonctionnel basse fréquence à $600 \mathrm{MHz}$ d'horloge, le vecteur de donnée étant celui qui est défini par la relation (10): trace du haut = sortie en division par 4 ; trace du bas = sortie multiplexée.

[Low frequence output signal at $600 \mathrm{Mbit} / \mathrm{s}$ data rate.] 
damment, par l'application de vecteurs de tests appropriés sur les accès. La fonction la plus critique des circuits étant celle de comptage, la mesure de la fréquence maximum de division permet d'obtenir les meilleures performances des multiplexeurs. Pour ces mesures, une seule voie de l'étage de multiplexage est ouverte par le vecteur des données, $\mathbf{E}=$ $(1,0,0,0)$. Remarquons que l'utilisation de sondes dynamiques spécifiques pourrait permettre une automatisation de ce type de mesure directement sur la plaquette de GaAs, afin de trier les puces (avant la découpe), sur la base de performances en haute fréquence.

Les tableaux I regroupent pour plusieurs échantillons de MUXV2 (en Ia) et MUX2 (en Ib) tous extraits d'une même plaquette ayant une tension de seuil moyenne de $-0,8 \mathrm{~V}$, les valeurs de la fréquence maximale $f_{\max }$, et de consommation $\boldsymbol{P}_{\mathrm{d}}$. $f_{\max }$ et $P_{\mathrm{d}}$ évoluent respectivement de 0,79 à $1,14 \mathrm{~W}$ et de 1330 à $1920 \mathrm{MHz}$. Cette dispersion de la fréquence maximum est liée d'une part aux variations de la tension de seuil sur la plaquette, et d'autre part, à l'importante sensibilité des bascules RST $\overline{\mathrm{T}} \mathrm{M} / \mathrm{E}$ aux horloges complémentaires. Rappelons en effet que pour une bascule à transfert sur front montant

Tableau I. - Résumé des performances de 5 échantillons du multiplexeur programmable (MUXV2) sur le tableau Ia et à 2 entrées fixes (MUX2) sur le tableau Ib.

\begin{tabular}{ccccc}
\hline $\begin{array}{c}\text { Numéro } \\
\text { de } \\
\text { circuit }\end{array}$ & $\begin{array}{c}\text { Consommation } \\
\text { (W) }\end{array}$ & \multicolumn{3}{c}{ Fréquence maximum } \\
$(\mathrm{MHz})$ & $\div 2$ & $\div 3$ & $\div 4$ \\
\hline $14 \mathrm{a}$ & 0,79 & 1330 & 1390 & 1550 \\
$12 \mathrm{a}$ & 0,86 & 1340 & 1440 & 1560 \\
$23 \mathrm{a}$ & 0,83 & 1460 & 1500 & 1600 \\
$18 \mathrm{a}$ & 0,95 & 1520 & 1610 & 1730 \\
$31 \mathrm{a}$ & 1,0 & 1570 & 1610 & 1760 \\
\hline
\end{tabular}

(a)

\begin{tabular}{ccc}
\hline $\begin{array}{c}\text { Numéro } \\
\text { de } \\
\text { circuit }\end{array}$ & $\begin{array}{c}\text { Consommation } \\
\text { (W) }\end{array}$ & $\begin{array}{c}\text { Fréquence } \\
\text { maximum } \\
(\mathrm{MHz})\end{array}$ \\
\hline $16 \mathrm{~b}$ & 0,95 & 1450 \\
$42 \mathrm{a}$ & 1,01 & 1850 \\
$42 \mathrm{~b}$ & 1,03 & 1850 \\
$41 \mathrm{~b}$ & 0,89 & 1900 \\
$10 \mathrm{a}$ & 1,14 & 1920 \\
\hline
\end{tabular}

(b)

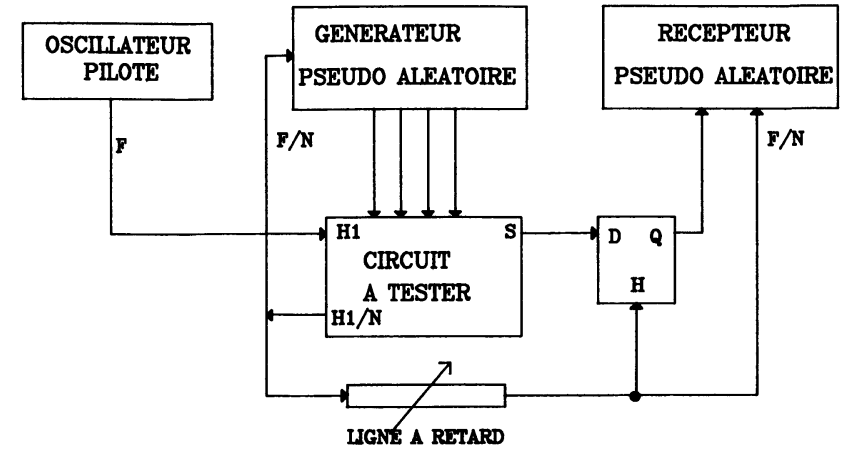

Fig. 11. - Dispositif expérimental de mesure de taux d'erreurs.

[Bit error rate measurement system.]

d'horloge, la marge de fonctionnement est limitée à un seul $T_{\text {pd }}$ en croisement à « 1 » et à $2 . T_{\text {pd }}$ en croisement à « 0 ». Dans le cas de quelques circuits mesurés, on observe que le croisement des horloges en dehors des marges précédentes, entrave la montée en fréquence. Cependant, les valeurs de $f_{\max }$ les plus élevées sont comparables aux fréquences maximums théoriques prévues en simulations, avec des écarts variant de 10 à $15 \%$; ces pourcentages d'erreurs étant par ailleurs du même ordre de grandeur que les erreurs relatives qui sont liées aux méthodes d'évaluation des capacités de couplage entre lignes sur les dessins de masque lors des simulations.

4.2.4 Sensibilité des circuits, durées des fronts d'ondes. - La sensibilité des multiplexeurs à l'horloge (ou à la donnée) est définie par l'amplitude de tension crête à crête minimale de l'horloge (ou de la donnée) pour laquelle les circuits continuent d'assurer les fonctions de comptage et de multiplexage attendues. Pour en effectuer les mesures, les circuits sont placés dans des conditions de fonctionnement qui mettent simultanément à contribution les différents blocs fonctionnels, toutes les sorties dynamiques étant chargées sur $50 \Omega$.

Pour un multiplexeur MUXV2 programmé sur 4 entrées, la figure 12a décrit la sensibilité à l'horloge en fonction du débit d'information en sortie ; celle-ci varie de 100 à $550 \mathrm{mV}$ c à c, entre 200 et $1550 \mathrm{Mbit} / \mathrm{s}$. La courbe de sensibilité à la donnée, du même circuit, est représentée sur la figure 13b ; celle-ci vaut $50 \mathrm{mV}$ c à c en moyenne, sauf aux faibles débits où elle remonte à $200 \mathrm{mV} \mathrm{c}$ à $\mathrm{c}$.

Les temps de montée $\left(t_{\mathrm{r}}\right)$ et de descente $\left(t_{\mathrm{f}}\right)$ des formes d'ondes en sortie ont été mesurées sur plusieurs échantillons, en fonction de la fréquence de multiplexage. A titre d'exemple, la figure 13 présente entre 700 et $1800 \mathrm{MHz}$, pour un multiplexeur à 2 entrées (MUX2), les variations de $t_{\mathrm{r}}$ et $t_{\mathrm{f}}$. Les valeurs passent de $190 \mathrm{ps}$ aux hautes fréquences à $350 \mathrm{ps}$ aux fréquences basses. 


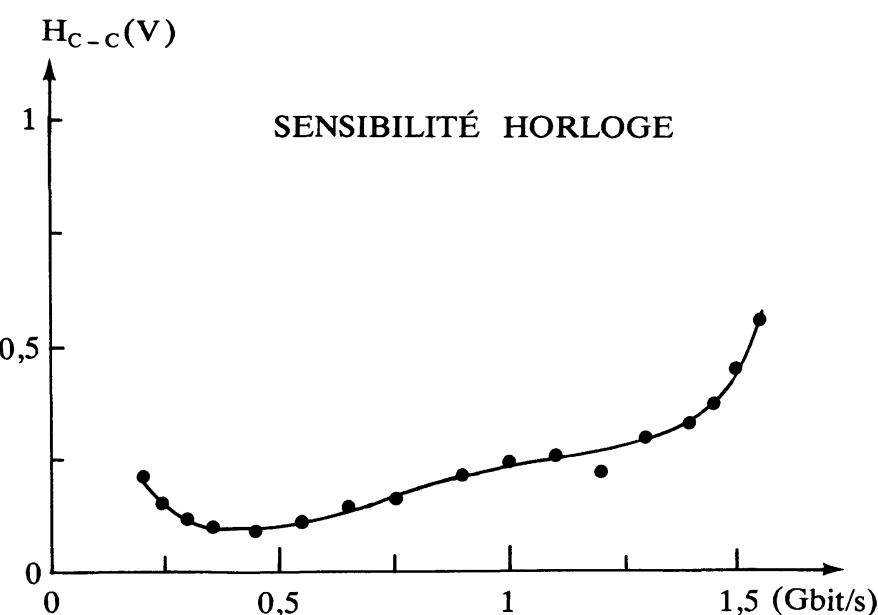

a)

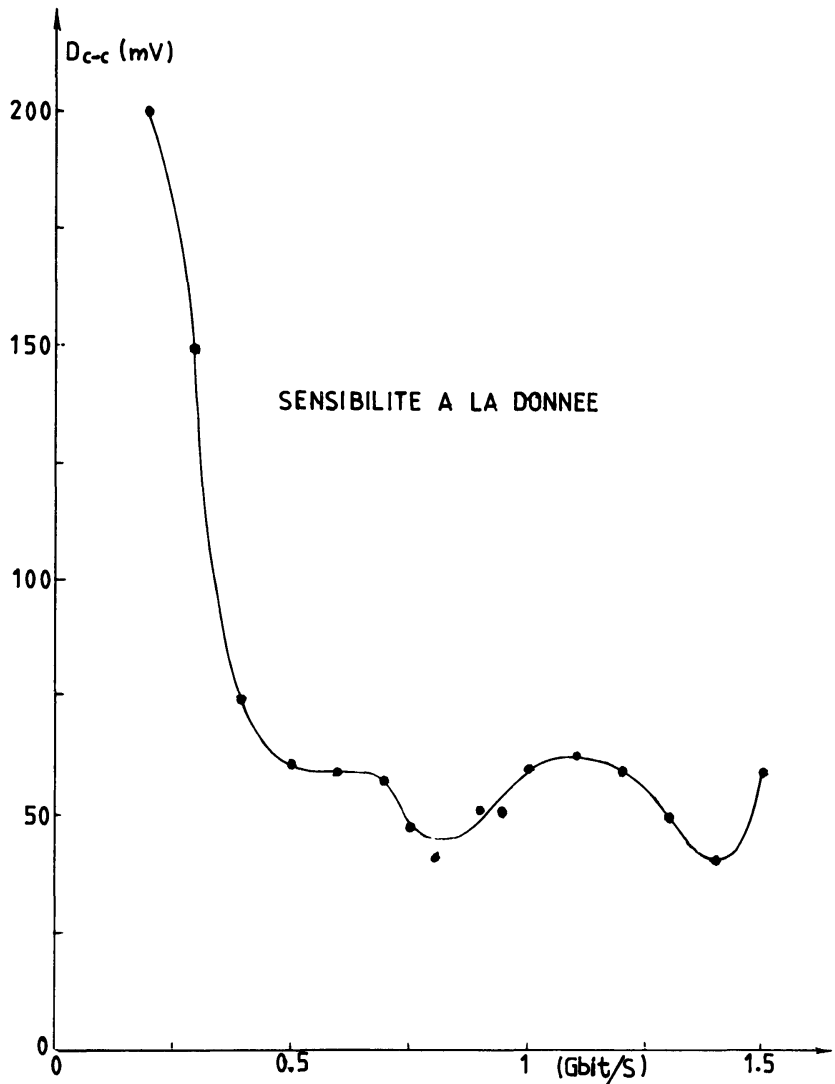

b)

Fig. 12. - Variations (a) de la sensibilité d'entrée horloge (b) de la sensibilité d'entrée de la donnée, en fonction du débit d'information à la sortie d'un multiplexeur programmable (MUXV2). $V_{\mathrm{DD}}=3,3 \mathrm{~V}, V_{\mathrm{Ss}}=-2,5 \mathrm{~V}$.

[Input sensitivity versus output data rate for a programmable multiplexer (a) clocking frequency (b) input data (peak to peak voltage).]

4.2.5 Mesures de taux d'erreurs. - Pour déterminer les performances des multiplexeurs dans les conditions d'utilisation effective (Fig. 1), une caractérisation plus complète des circuits consistant à simuler

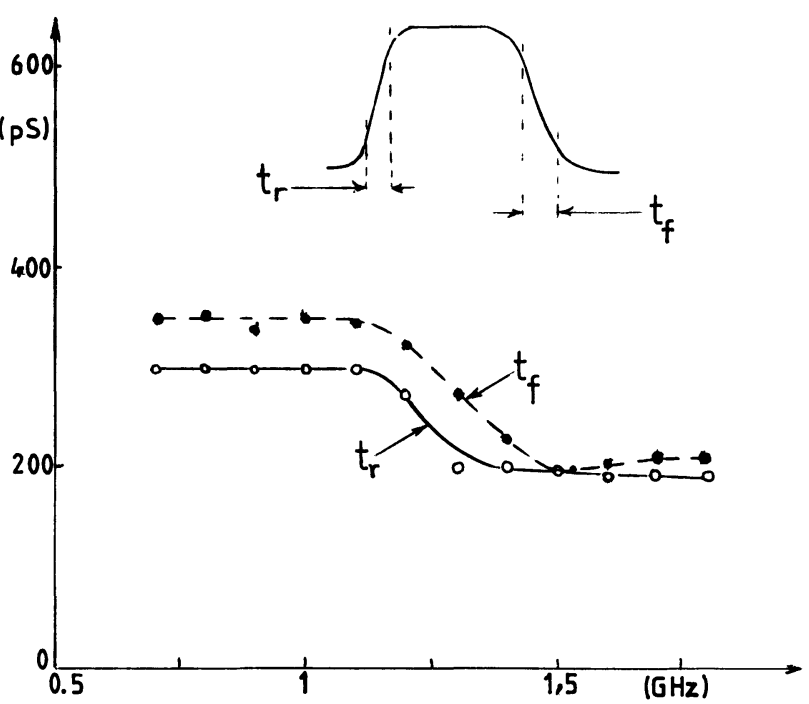

Fig. 13. - Variations en fonction de la fréquence de multiplexage, des temps de montée $\left(t_{\mathrm{r}}\right)$ et de descente $\left(t_{\mathrm{f}}\right)$ des signaux de sortie d'un multiplexeur à 2 entrées (MUX2). $V_{\mathrm{DD}}=3,3 \mathrm{~V}, V_{\mathrm{ss}}=-2,5 \mathrm{~V}$.

[Rise $\left(t_{\mathrm{r}}\right)$ and fall $\left(t_{\mathrm{f}}\right)$ times versus operating frequency of a 2 inputs multiplexer.]
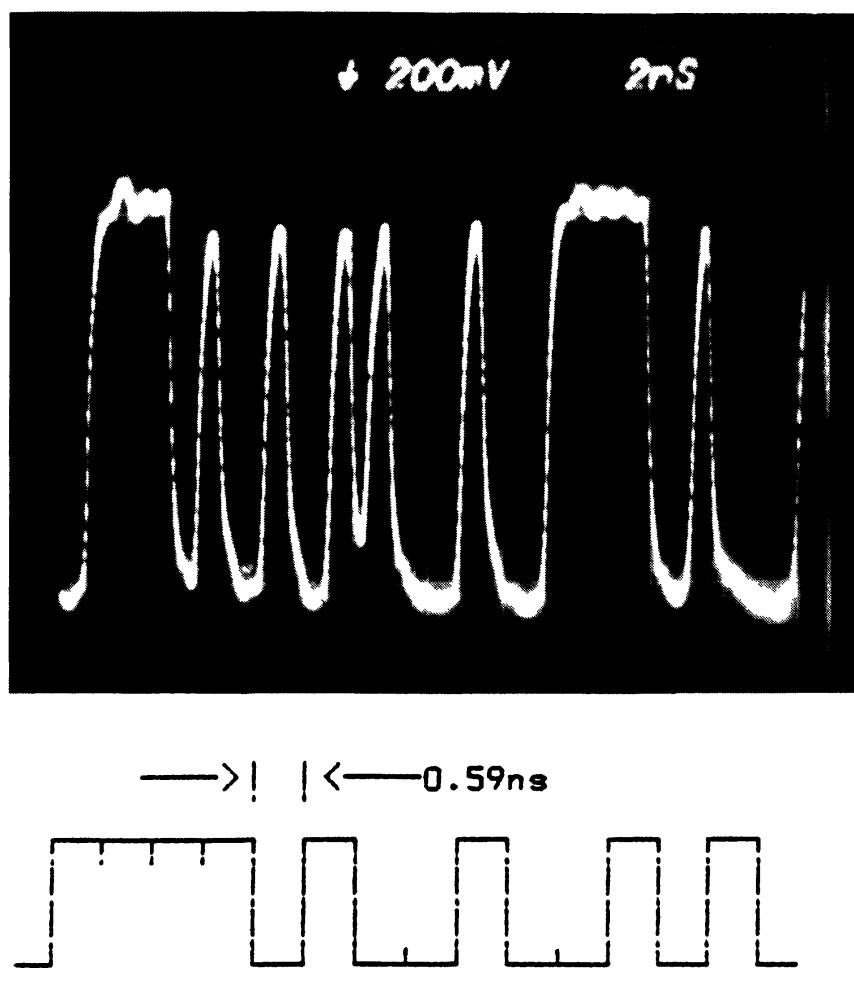

1111011001

Fig. 14. - Photographie du signal à $1,7 \mathrm{Gbit} / \mathrm{s}$ correspondant au multiplexage de $3 \times 560 \mathrm{Mbit} / \mathrm{s}$ (multiplexeur programmé sur 3 entrées).

[Multiplexing output data at $1.7 \mathrm{Gbit} / \mathrm{s}$ resulting from $3 \times$ $560 \mathrm{Mbit} / \mathrm{s}$ inputs data sequences.] 
des conditions de fonctionnement proches de l'utilisation système a été effectuée. Pour ce faire on utilise le dispositif expérimental représenté sur la figure 11. Pour une fréquence pilote $f$, les $n$ entrées du multiplexeur sont alimentées par des signaux numériques pseudo-aléatoires, tandis que le signal série résultant du multiplexage est sous-échantillonné par une bascule de type $\mathrm{D}$ à la fréquence $f / n$. L'analyse du sous-échantillon fournie par cette bascule à l'aide d'un récepteur pseudo-aléatoire permet alors, lorsque toutes les fonctions intégrées du multiplexeur sous test sont remplies, de retrouver un des signaux d'entrée parmi $n$, avec un taux d'erreur qui doit être nul. La commande de phase (ligne à retard) de l'horloge d'échantillonnage permet d'explorer successivement les $n$ entrées.

Afin d'aboutir à une sélection de circuits pouvant satisfaire des applications systèmes, les taux d'erreurs n'ont été recherchés que sur les puces ayant présenté les meilleurs résultats après les mesures décrites dans les paragraphes précédents.

Les circuits à tester sont placés en fonctionnement nominal (niveaux horloges et données suffisants. Séquences de données pseudo-aléatoires égaux à $2^{k}-1$, avec $k=11$ ) sur une durée permettant à la puce de traiter plusieurs dizaines de gigabits. L'ensemble des échantillons présélectionnés par des tests dynamiques ont fourni des taux d'erreurs nuls.
La figure 14 présente en exemple, la photographie d'un signal typique à $1,7 \mathrm{Gbit} / \mathrm{s}$, fournie dans les conditions de mesure de taux d'erreurs, par un multiplexeur programmé sur 3 entrées, qui reçoit 3 trains numériques à $560 \mathrm{Mbit} / \mathrm{s}$ chacun.

\section{Conclusion.}

Des multiplexeurs haut débit à rang fixe ou programmable ont été conçus et réalisés en logique BFL à grille de $1 \mu \mathrm{m}$. De complexité moyenne ces circuits ont été simulés en régime temporel non linéaire dans ASTEC3 selon une méthode de découpage en blocs fonctionnels quasi-indépendants. Leurs performances en débit numérique est située dans la gamme de 1,5 à $2 \mathrm{Gbit} / \mathrm{s}$, pour une consommation moyenne autour du watt. Malgré l'absence des tests de fiabilité, les résultats de mesures simulant un fonctionnement réel ont montré leur aptitude à fonctionner de manière satisfaisante à haut débit dans une application système. D'autre part, les résultats obtenus nous confirment dans l'idée qu'un circuit numérique sur GaAs doit intégrer une fonction complète sur la puce pour éviter les problèmes liés à l'assemblage de circuits standards, dont les connexions apportent des délais supplémentaires, et dont les étages de sortie accroissent la consommation globale.

\section{Bibliographie}

[1] Greiling, P. T., High-speed digital IC performance outlook. IEEE Trans. Microwave Theory Tech. MTT-35 (1987) 245-259.

[2] Osafune, K., OhwAdA, K., Ultra-high-speed GaAs monolihic prescaler and phase frequency comparator IC. IEEE Trans. Microwave Theory Tech. MTT-34 (1986) 786-790.

[3] Henry, M., Maheo, P., Thebault, C., Vergos, J., 1,7 Gbit/s multiplexing-demultiplexing system for a high-bit-rate optical link, Electron. Lett. 21 (1985) 487-489.

[4] Azizi, C., Kamdem, J., Graffeuil, J., Rossel, P., Computer simulations of non linear GaAs MESFET circuits, 6th ESSCIRC 80, Univ. Scientifique et Médicale (Grenoble, France) septembre 1980.

[5] Kamdem, J., Maimouni, R., Azizi, C., Graffeuil, J., Rossel, P., Méthodologies d'étude expérimentale et de conception assistée par ordinateur des amplificateurs de puissance à TEC GaAs, Revue Phys. Appl. 18 (1983) 213-227.

[6] Liechti, C. A. et al., A GaAs MSI word generator operating at $5 \mathrm{Gbits} / \mathrm{s}$ data rate, IEEE Trans. Microwave theory Tech. MTT-30 (1982) 9981006.

[7] Jarry, J., Gloanec, M., CAMPS, P., Encapsulation des circuits intégrés numériques GaAs utilisés en logique subnanoseconde, IVième Journée Nationales Microondes (Lannion, France) 1984.

[8] Kamdem, J., Henry, M., Klein, J. P., AleXandre, F., GloANEc, M., Cryogenic GaAs integrated circuits using a lightly doped GaAs FET structure, High-speed Electronics, Proc. (Stockholm, Sweden) 1986 172-176.

[9] Rossel, P., Azizi, C., Graffeuil, J., Modèles du transistor à effet de champ à l'arséniure de gallium, Rev. polytechnique 1372-4-78 (1977) 335-345.

[10] Kamdem, J., Méthodologies de conception des circuits intégrés logiques GaAs, Communication aux journées d'études SEE (Trégastel, France) juin 1987.

[11] Rossel, P., Azizi, C., Tranduc, H., Graffeuil, J., SAutereau, J. F., Modèles physiques pour la conception des fonctions logiques à l'arséniure de gallium, Rapp. de contrat DGRST CCM 76-7802 (1978).

[12] Suyama, K., Kusakawa, H., Okamura, S., FuKUTA, M., An MSI GaAs integrated circuit : 4 bit arithmetic and logic unit, IEEE J. SolidState Circuits sc-17 (1982) 653-657.

[13] IshiI, Y., INo, M., IDA, M., Hirayama, M., OHMORI, M., Processing technologies for GaAs Memory LSIs, GaAs IC Symp. (1984) 121-124. 
[14] Welbourn, A. D., Blau, G. L., Livingstone, A. W., A high speed GaAs 8 bit multiplexer using capacitor-coupled logic, IEEE J. SolidState Circuits sc-18 (1983) 359-364.

[15] Nakamura, H., TANAKa, K., INOKUChI, K., SAITo, T., Kawakami, Y., Sano, Y., AkiYama, M., KAMINISHI, K., 2 GHz multiplexer and demultiplexer using DCFL/SBFL circuit and the precise $V_{\text {th }}$ control process, GaAs IC Symp. (1986) 151154.

[16] Flahive, P. G., Clemetson, W. J., O’Connor, P., Dori, A., Schunt, S. C., A GaAs DCFL chip set for multiplex and demultiplex applications at Gigabit/s data rates, GaAs IC Symp. (1984) 710.
[17] Takada, T., Nozawa, K., IdA, M., Asai, K., A Giga-bit-rate GaAs multi-functional LSI with half-micron gate buried p-layer FET's, GaAs IC Symp. (1986) 7-10.

[18] REIN, H. M., REIMANN, R., 6 Gbit/s multiplexer and regenerating demultiplexer ICs for optical transmission systems based on a standard bipolar technology, Electron. Lett. 22 (1986) 988-990.

[19] Hasegawa, K., Tezuka, A., Uenoyama, T., NisHII, K., BANDO, K., UTSUMI, K., ONUMA, T., High yield and low power multiplexer/demultiplexer by SCFL, GaAs IC Symp. (1986) 147-150. 